\title{
Imaging and Computer Vision Resources
}

This appendix contains a list of some resources for computer vision and imaging, including commercial products, open-source projects, organizations, and standards bodies.

\section{Commercial Products}

\begin{tabular}{|c|c|}
\hline Name & Matlab \\
\hline Description & $\begin{array}{l}\text { Industry standard math package with many scientific package } \\
\text { options for various fields including imaging and computer } \\
\text { vision. Includes a decent software development environment, } \\
\text { providing add-on libraries for computer vision, image processing, } \\
\text { visualization, more. Suited well for code development. }\end{array}$ \\
\hline Library API & Extensive API libraries Internal to the SDE. \\
\hline SDE & Includes software development environment for coding. \\
\hline Open Source & Not for the product, but possibly for some code developed by users. \\
\hline Link & http://www.mathworks.com/products/matlab/ \\
\hline Name & Mathematica \\
\hline Description & $\begin{array}{l}\text { Industry standard math package with many scientific package } \\
\text { options for various fields, including image processing and computer } \\
\text { vision. Excellent for creation of publication-ready visualizations } \\
\text { and math notebooks. Add-on libraries for computer vision, image } \\
\text { processing, visualization, more. }\end{array}$ \\
\hline Library API & Extensive API libraries Internal to the SDE. \\
\hline SDE & $\begin{array}{l}\text { Includes a default function-based script development environment, } \\
\text { and some code development add-ons. }\end{array}$ \\
\hline Open Source & Not for the product, but possibly for code developed by users. \\
\hline Link & http://wWw.wolfram.com/mathematica/ \\
\hline
\end{tabular}




\begin{tabular}{ll}
\hline Name & Intel TBB, Intel IPP, Intel CILK++ \\
\hline Description & $\begin{array}{l}\text { Intel provides libraries, languages, and compilers optimized for } \\
\text { the IA instruction set. Intel TBB is a multi-threading library for } \\
\text { single and multi-core processors, Intel IPP provides imaging and } \\
\text { computer vision performance primitives optimized for IA and } \\
\text { SIMD instructions and in some cases GPGPU, and Intel CILK++ is a } \\
\text { language for writing SIMD/SIMT parallel code. }\end{array}$ \\
& Extensive API libraries. \\
Library API & No, but Intel CILK++ is a programming language. \\
SDE & No. \\
Open Source & http://software.intel.com/en-us/intel-tbb \\
Link & http://software.intel.com/en-us/intel-ipp \\
\end{tabular}

\section{Open Source}

\begin{tabular}{ll}
\hline Name & OpenCV \\
\hline Description & $\begin{array}{l}\text { Industry standard computer vision and image processing library, } \\
\text { used worldwide by major corporations and others. }\end{array}$ \\
Library API & Extensive API library. \\
SDE & No. \\
Open Source & BSD license. \\
Link & http://opencv.org/ \\
\hline Name & ImageJ - FIJI \\
\hline Description & $\begin{array}{l}\text { Application for image processing, visualization, and computer } \\
\text { vision. Developed by the USG National Institutes of Health[502], } \\
\text { available for public use. Extensive. FIJI is a distribution of ImageJ } \\
\text { with many plug-ins submitted by the user community. }\end{array}$ \\
Library API & $\begin{array}{l}\text { No. } \\
\text { SDE }\end{array}$ \\
No. \\
Open Source & $\begin{array}{l}\text { Public domain use. } \\
\text { http://rsbweb.nih.gov/ij/index.html } \\
\text { http://rsb.info.nih.gov/ij/plugins/ } \\
\text { http://fiji.sc/Fiji }\end{array}$ \\
\hline
\end{tabular}




\begin{tabular}{|c|c|}
\hline Name & VLFEAT \\
\hline Description & $\begin{array}{l}\text { C library containing a range of common computer vision algorithms } \\
\text { for feature description, pattern matching, and image processing. }\end{array}$ \\
\hline Library API & Extensive API library. \\
\hline SDE & No. \\
\hline Open Source & BSD license. \\
\hline Link & http://vlfeat.org \\
\hline Name & VTK \\
\hline Description & $\begin{array}{l}\text { C++ library containing a range of common image processing, } \\
\text { graphics, and data visualization functions. Includes GUI widgets. } \\
\text { VTL also provides consulting. }\end{array}$ \\
\hline Library API & Extensive API library. \\
\hline SDE & No. \\
\hline Open Source & BSD license. \\
\hline Link & http://vtk.org/ \\
\hline Name & Meshlab \\
\hline Description & $\begin{array}{l}\text { Application for visualizing, rendering, annotating, and converting } \\
\text { 3D data meshes such as point clouds and CAD designs. Extensive. } \\
\text { Uses the VCG library from ISTI - CNR. }\end{array}$ \\
\hline Library API & No. \\
\hline SDE & No. \\
\hline Open Source & BSD license. \\
\hline Link & http://meshlab.sourceforge.net/ \\
\hline Name & PfeLlb \\
\hline Description & Library for image processing and computer vision acceleration. \\
\hline Library API & Yes. \\
\hline SDE & No. \\
\hline Open Source & No. \\
\hline Link & See reference[495]. \\
\hline
\end{tabular}




\begin{tabular}{|c|c|}
\hline Name & Point Cloud Library (PCL) \\
\hline Description & $\begin{array}{l}\text { Extensive open-source library for dealing primarily with 3D point } \\
\text { clouds, including implementations of many cutting-edge 3D descriptors } \\
\text { from the latest academic research and visualization methods. }\end{array}$ \\
\hline Library API & Yes. \\
\hline SDE & No. \\
\hline Open Source & Yes. \\
\hline \multirow[t]{3}{*}{ Link } & http://pointclouds.org/downloads/ \\
\hline & http://pointclouds.org/documentation/ \\
\hline & http://docs.pointclouds.org/trunk/a02944.html \\
\hline Name & Shogun Machine Learning Toolbox \\
\hline Description & Library for machine learning and pattern matching. Extensive. \\
\hline Library API & Yes. \\
\hline SDE & No. \\
\hline Open Source & GPL. \\
\hline Link & http://shogun-toolbox.org/page/features/ \\
\hline Name & Halide High-Performance Image Processing Language \\
\hline Description & C++ language classes optimized for SIMD, SIMT, and GPGPU. \\
\hline Library API & Yes. \\
\hline SDE & No. \\
\hline Open Source & Open-source MIT license. \\
\hline Link & http://halide-lang.org/ \\
\hline Name & REIN (Recognition INfrastructure) Vision Algorithm Framework \\
\hline Description & $\begin{array}{l}\text { Framework for computer vision in robotics; uses ROS operating system. } \\
\text { See references }[397,503] \text {. }\end{array}$ \\
\hline Library API & Yes. \\
\hline SDE & No. \\
\hline Open Source & Open-source MIT license. \\
\hline Link & http://wiki.ros.org/rein \\
\hline
\end{tabular}




\begin{tabular}{ll}
\hline Name & ECTO -Graph Network Construction for Computer Vision \\
\hline Description & $\begin{array}{l}\text { Library for creating directed acyclic graphs of functions for computer } \\
\text { vision pipelines, supports threading. Written in a C++/Python } \\
\text { framework. Can integrate with OpenCV, PCL and ROS. }\end{array}$ \\
Library API & Yes. \\
SDE & No. \\
Open Source & Apparently. \\
Link & http://plasmodic.github.io/ecto/ \\
\hline
\end{tabular}

\section{Organizations, Institutions, and Standards}

\section{Microsoft Research}

http://academic.research.microsoft.com/

\section{CIE}

http://www.cie.co.at/

\section{ICC}

http://www.color.org/index.xalter

\author{
CAVE Computer Vision Laboratory \\ http://Www.cs.columbia.edu/CAVE/
}

RIT Munsel Color Science Laboratory

http://www.rit.edu/cos/colorscience/
Microsoft Research has one of the largest staff of computer vision experts in the world, and actively promotes conferences and research. Provides several good resources online.

International Commission on

Illumination, abbreviated CIE after the French name, provides standard illuminant data for a range of light sources as it pertains to color science, as well as standards for the well-known color spaces CIE XYZ, CIE Lab and CIE Luv.

International Color Consortium provides the ICC standard color profiles for imaging devices, as well as many other industry standards, including the sRGB color space for color displays.

Computer Vision Laboratory at Columbia University, directed by Dr. Shree Nayar, provides world-class imaging and vision research.

Rochester Institute of Technology Munsel Color Science Laboratory is among the leading research institutions in the area or color science and imaging, provides a wide range of resources, and has with strong ties to industry imaging giants such as Kodak, Xerox, and others. 


\section{OPENVX KHRONOS}

http://Www. khronos.org/openvx

\section{SPIE}

Society for Optics and Photonics

Journal of Medical Imaging

Journal of Electronic Imaging

Journal of Applied Remote Sensing

http://spie.org/

\section{IEEE}

CVPR, Computer Vision and Pattern Recognition

PAMI, Pattern Analysis and Machine Intelligence

ICCV, International Conference on

Computer Vision

IP, Trans. Image Processing

http://ieee.org

\section{CVF}

Computer Vision Foundation

http://www.cv-foundation.org/

\section{NIST - Image Group (USG)}

National Institute Of Standards

http://www.nist.gov/itl/iad/ig/

\section{I20 - Darpa information innovation office (USG)}

http://www.darpa.mil/

Our_Work/I20/Programs/

http://www.darpa.mil/

OpenCatalog/index.html
OPENVX is a proposed standard for lowlevel vision primitive acceleration, operated with the KHRONOS standards group.

Interdisciplinary approach to the science of light, including photonics, sensors, and imaging; promotes conferences, publishes journals.

Society for publication of journals and conferences, including various computer vision and imaging topics.

Promotes computer vision, provides dissemination of papers.

Promotes computer vision and imaging grand challenges; covers biometrics standards, fingerprint testing, face, iris, multimodal testing, next generation test bed.

Extensive array of computer vision and related program research for military applications.

Some work is released to the public via the OpenCatalog. 


\section{Journals and Their Abbreviations}

CVGIP Graphical Models /graphical Models and Image

Processing / computer Vision, Graphics, and Image Processing

CVIU Computer Vision and Image Understanding

IJCV International Journal of Computer Vision

IVC Image and Vision Computing

JMIV Journal of Mathematical Imaging and Vision

MVA Machine Vision and Applications

TMI - IEEE Transactions on Medical Imaging

\section{Conferences and Their Abbreviations}

3DIM International Conference on 3-D Imaging and Modeling 3DPVT 3D Data Processing Visualization and Transmission ACCV Asian Conference on Computer Vision AMFG Analysis and Modeling of Faces and Gestures

BMCV Biologically Motivated Computer Vision

BMVC British Machine Vision Conference

CRV Canadian Conference on Computer and Robot Vision

CVPR Computer Vision and Pattern Recognition

CVRMed Computer Vision, Virtual Reality and Robotics in Medicine

DGCI Discrete Geometry for Computer Imagery

ECCV European Conference on Computer Vision

EMMCVPR Energy Minimization Methods in Computer Vision and Pattern Recognition

FGR IEEE International Conference on Automatic Face and Gesture Recognition

ICARCV International Conference on Control, Automation, Robotics and Vision

ICCV International Conference on Computer Vision

ICCV Workshops

ICVS International Conference on Computer Vision Systems 
ICWSM International Conference on Weblogs and Social Media

ISVC International Symposium on Visual Computing

NIPS Neural Information Processing Systems

Scale-Space Scale-Space Theories in Computer Vision

VLSM Variational, Geometric, and Level Set Methods in

Computer Vision

WACV Workshop on Applications of Computer Vision

\section{Online Resources}

\begin{tabular}{|c|c|}
\hline Name & CVONLINE \\
\hline Description & $\begin{array}{l}\text { Huge list of computer vision software and projects, indexed } \\
\text { to Wikipedia }\end{array}$ \\
\hline Link & http://homepages.inf.ed.ac.uk/rbf/CVonline/environ.htm \\
\hline Name & Annotated Computer Vision Bibliography \\
\hline Description & $\begin{array}{l}\text { Huge index of links to computer vision topics, references, } \\
\text { software, more }\end{array}$ \\
\hline Link & http://www.visionbib.com/bibliography/contents.html \\
\hline Name & NIST Online Engineering Statistics Handbook (USG) \\
\hline Description & Handbook for statistics, includes examples and software \\
\hline Link & http://www.itl.nist.gov/div898/handbook/ \\
\hline Name & The Computer Industry (David Lowe) \\
\hline Description & $\begin{array}{l}\text { Includes links to major computer vision and imaging product } \\
\text { companies }\end{array}$ \\
\hline Link & http://www.cs.ubc.ca/ lowe/vision.html \\
\hline
\end{tabular}

